\title{
A Prospective Study about the Influence of Selenium Based Supplements on the Autoimmune Process Evolution and the Health-Related Quality of Life in Patients with Chronic Autoimmune Thyroiditis
}

\author{
Maximilian Cosma Gliga ${ }^{\star *}$, Ionela Maria Pascanu1², Camelia Gliga1,3, Ancuta Elena Zahan¹, Iulian \\ Merlan ${ }^{1}$ \\ 1. Endocrinology Clinic, County Clinical Hospital Mureș, Romania \\ 2. Endocrinology department of University of Medicine, Pharmacy, Sciences and Technology of Târgu Mures, Romania \\ 3. Histology department of University of Medicine, Pharmacy, Sciences and Technology of Târgu Mures, Romania
}

\begin{abstract}
Objective: The purpose of this study was to investigate the benefits of two different Selenium based supplements on patients with chronic autoimmune thyroiditis. Methods: We conducted a prospective study on 50 patients with chronic autoimmune thyroiditis, who were divided into three different treatment groups, one group taking Selenium $100 \mu \mathrm{g}$, one Procor T (a combination of Selenium $100 \mu \mathrm{g}$ and other elements like copper, Zinc and Q10 Conenzyme) and one control group taking Placebo pills. We measured on two follow up visits the antibody levels (anti-thyroidperoxidase- TPO Ab) and offered each patient a standardised questionnaire regarding the thyroid-related quality of life (THYPROro). Results: At the 6 months follow up visit there was a statistically significant decrease in the antibody levels for each treatment group compared to the base levels. The THYPROro questionnaire scores showed an improvement in most aspects regarding the quality of life as well, but there was no significant difference between the placebo and the treated groups in the magnitude of this improvement. Conclusions: Based on our results, we could not identify a certain benefit in improving quality of life with the supplementation of Selenium, as the improvements were at a similar level for the patients who took Placebo pills. Further studies with more patients, as well as taking the Selenium defficiency in consideration (by measuring the basal serum level of Selenium for each patient) would be required to find the target group of patients who could have most benefits of Selenium-based supplementation.
\end{abstract}

Keywords: chronic autoimmune thyroiditis, selenium, quality of life, autoantibodies, hashimoto

Received 20 February 2019 / Accepted 16 May 2019

\section{Introduction}

Chronic autoimmune thyroidits is one of the most common diseases diagnosed in endocrinological practice, and is also the main cause of hypothyroidism in adults. Patients with this disease present a broad spectrum of symptoms and signs, ranging from mild to severe, which can be caused both by the thyroid dysfunction (ussualy hypothyroidism) but also by unknown mechanisms through the autoimmune process itself. It is not uncommon for patients with normal thyroid function to manifest different types of symptoms, which affect the quality of life in various ways [1,2]. Selenium is an element with multiple roles in the human body homeostasis, even though most of it's mechanisms of actions are still unknown [3, 4]. Selenium is known to have a role in the production of Selenoproteins, one of these proteins having a major role in the methabolism of thyroid hormones- iodotyronine deiodinase. This enzymes have an important role in the activation of thyroid hormones, by converting the prohormone thyroxine (T4) to the more active form triiodothyronine (T3) [5].The objective of our study was to investigate the effects of two different types of selenium-based supplements on the quality of life (measured through a standardised ques-

* Correspondence to: Maximilian Cosma Gliga

E-mail: max_mg_tenn@yahoo.com tionnaire) and the serum anti-thyroidperoxidase antibody levels (TPO-Ab) in patients diagnosed with chronic autoimmune thyroidits having a normal thyroid function with or without Levothyroxine (LT4) therapy.

\section{Methods}

Our study started in 2016 in the Endocrinology compartment of the Mures County Hospital. Durine a timeframe of approximatly one and a half year 50 patients were enrolled in the study. Before starting the study we obtained an approval form from the ethics comitee of the University of Medicine and Pharmacy of Targu Mures (annexed). Every patient who entered the study signed an informed consent form which contains a detailed description of the study design and implications. The inclusion criteria were the following: patients diagnosed with autoimmune thyroidits (based on elevated anti-thyroid autoantibodies- TPOAbor anti- $\mathrm{Tg} \mathrm{Ab}$ ) and/or sonographic aspect typical of autoimmune thyroidits; patients with normal thyroid function with or without thyroid hormone substitution(thyroid stimulation hormone- TSH and thyroxine-FT4 hormone within normal reference range). For the laboratory measurements mentioned chemiluminescence assay was used (Architect I 1000 SR Immunoassay).The reference ranges for each assay were the following: TSH: $0.35-4.94 \mu \mathrm{IU} / \mathrm{ml}$, fT4: 0.7-1.48 ng/ml, TPOAb: 0-5.61 IU/ml, TgAb: 
$0-4.11 \mathrm{IU} / \mathrm{ml}$. The exclusion criteria were set as following: previous diagnosis of other thyroid diseases; history of radioactive iodine treatment, anti-thyroid medication or other therapies that could influence thyroid function; patients with other associeted severe pathology which could significantly influence the health-related quality of life; patients uncapable of adherence to the study for various reasons or who did not sign the informed consent. We divived the patients into three treatment groups through a randomisation and blinding process (trail drugs were labeled as A, B and C, with the exact supplement contained being revealed only at the end of the study to the authors): one group treated with Selenium $100 \mu \mathrm{g}$ one tablet/day, the second group treated with a supplement called Procor $\mathrm{T}$ (a combination of Selenium $100 \mu \mathrm{g}$, Q10 coenzyme, Copper and Zync) one tablet/day, and the last group received Placebo-type tablets (tablets containing no active substances). All the mentioned trial drugs were provided by the company Sunwave Pharma. We did not lose any patient during follow-up. For the evaluation methods and the gathering of informations and data, we conducted an initial visit and two follow-up visits after 3 and 6 months. In all three visits the following investigations were conducted: anamnesis, clinical exam, TPO-Ab , FT4 and TSH serum levels, thyroid ultrasound and data obtained from the filling of the standardised Thyroid-related quality of life questionnaire (THYPROro). This instrument called THYPROro is a questionnaire made for evaluating the impact of chronic thyoird diseases on health-related quality of life. It was first created in Denmark by Dr. Torquil Watt and colaborators from Ringhospitalet University Hospital of Copenhagen [6]. Following several international studies this instrument was validated and translated into Romanian language[7]. The questionnaire contains a total of 85 questions grouped into 13 scales which cover the following: physical symptoms, mental symptoms, general well being, social life and one final question regarding quality of life as a whole. The patients can answer each question by themselves with choosing one option from 0 (none) to 4 (very much) after a short introduction. A final score will be calculated for each scale which can range from 0 to 100 ( the higher the score the greater the negative impact on the quality of life). For the analysis of the statistical difference between the means of different groups we used the nonparametric tests Friedman and Mann-Whitney. We set the level of statistical significance as the standard $\mathrm{p}=0.05$.

\section{Results}

The final size of each treatment groups was set as following: Placebo group 16 patients, Selenium group 17 patients and Procor T group 17 patients. All patients included had a normal thyroid function (FT4 and TSH within reference range) with $60 \%$ of the patients being under LT4 treatment and $40 \%$ with no hormonal treatment. $72 \%$ of the patients included had a ultrasound aspect with nodules, while $28 \%$ did not present any nodules. The TPOAb anti- bodies were positive in $88 \%$ of the patients and $22 \%$ of the patients had positive values for $\mathrm{TgAb}$ (Table I).

Regarding the results of the THYPROro scores for each treatment group on all three visits, there was a statistically significant decrease (meaning an improvement in the quality of life) on most scales for all three treatment groups, however no statistically significant difference between the magnitude of this improvement when comparing each treated group with the Placebo group. ( $p>0.05$ for all scales) (Table II).

By analysing the results regarding the TPOAb values we noticed there was a decrease of antibody levels in all three treatment groups, but the decrease proved to be statistically significant only for the group Procor $\mathrm{T}$ and Placebo (p $<0.05$ ). We also applied a Mann-Whitney test to compare the difference of the TPOAb levels between the entry- and 6 months visit between the Placebo group and each treatment group, with the results showing no statistically significant difference in both cases (Placebo and ProcorT $: \mathrm{p}$ $=0.95$, Placebo and Selenium: $\mathrm{p}=0.09)$ (Figure 1).

The results of the THYPROro scores from the last question of the questionnaire about the general impact of the thyroid disease on the quality of life showed a similar pattern to the TPOAb, with a tendency to decrease, the difference being statistically significant only for the Procor $T$ and Placebo groups $(\mathrm{p}=0.004$ and 0.0003$)$ but not for Selenium group $(\mathrm{p}=0.46)$ (Figure 2$)$.

The TSH levels did not show any statistically significant difference between the three visits on none of the treatment groups. (We applied Friedman test for assessing this aspect $-\mathrm{p}>0,05$ for every treatment group) (Figure 3).

\section{Discussions}

Some research have demonstrated that Selenium has an influence on the differentiation process of the lymphocyte $\mathrm{T}$ cells. Therefore increasing Selenium serum levels might produce a raise in the Ly $\mathrm{T}$ supressor cell population, which would lead to a decrease in the inflammatory infiltration of the thyroid and the production of autoan-

Table I. General characteristics of the patients group (DS= standard deviation)

\begin{tabular}{lc}
\hline Group size: & 50 \\
Women & $49(98 \%)$ \\
Men & $1(2 \%)$ \\
Average age & $51 \pm 11.72 \mathrm{SD}$ \\
Patients under LT4 treatment & $30(60 \%)$ \\
Patients without LT4 treatment & $20(40 \%)$ \\
Nodular ultrasound aspect & $14(28 \%)$ \\
Nodule-free ultrasound aspect & $36(72 \%)$ \\
TPO Ab positive & $44(88 \%)$ \\
TgAb positive & $11(22 \%)$ \\
Average height & $163 \mathrm{~cm} \pm 6.40 \mathrm{SD}$ \\
Average weight & $72 \mathrm{~kg} \pm 15.58 \mathrm{SD}$ \\
Average BMI (Body Mass Index) & $27 \pm 5.67 \mathrm{SD}$ \\
Normal weight patients & $23(46 \%)$ \\
Overweight patients (BMI 25-30) & $12(24 \%)$ \\
Grade 1 obesity patients (BMI 30-35) & $10(20 \%)$ \\
Grade 2 obesity patients (BMI 35-40) & $5(10 \%)$ \\
\hline
\end{tabular}


Table II: THYPROro average scores for each treatment group on all three visits.

\section{PROCOR T GROUP}

\begin{tabular}{|c|c|c|c|c|}
\hline Scale & First screening visit & 3 months- follow-up visit & 6 months- follow-up visit & $\mathrm{p}^{*}$ \\
\hline Goiter symptoms & 34.75 & 17.11 & 16.97 & 0.00 \\
\hline Hypethyroidism symptoms & 35.15 & 22.02 & 17.64 & 0.01 \\
\hline Hypothyroidism symptoms & 44.11 & 30.51 & 22.05 & 0.00 \\
\hline Eye symptoms & 22.80 & 15.62 & 12.68 & 0.02 \\
\hline Fatigue & 52.73 & 45.79 & 46.85 & 0.08 \\
\hline Cognitive problems & 31.86 & 25.98 & 23.28 & 0.41 \\
\hline Anxiety & 47.54 & 27.69 & 23.04 & 0.00 \\
\hline Emotional disturbances & 45.75 & 32.18 & 31.37 & 0.001 \\
\hline Social life & 15.80 & 10.66 & 12.13 & 0.4 \\
\hline Personal life & 24.75 & 13.23 & 13.23 & 0.004 \\
\hline Sexual problems & 36.02 & 29.41 & 33.80 & 0.479 \\
\hline Cosmetic problems & 20.34 & 17.40 & 8.08 & 0.114 \\
\hline \multicolumn{5}{|l|}{ PLACEBO GROUP } \\
\hline Goiter symptoms & 47.98 & 23.43 & 20.45 & 0.001 \\
\hline Hypethyroidism symptoms & 38.67 & 19.72 & 19.92 & 0.002 \\
\hline Hypothyroidism symptoms & 47.65 & 31.25 & 22.65 & 0.001 \\
\hline Eye symptoms & 35.35 & 17.58 & 17.38 & 0.002 \\
\hline Fatigue & 54.24 & 43.08 & 45.98 & 0.001 \\
\hline Cognitive problems & 43.80 & 26.30 & 24.74 & 0.004 \\
\hline Anxiety & 48.17 & 27.86 & 28.65 & 0.004 \\
\hline Depression & 52.67 & 36.38 & 32.58 & 0.007 \\
\hline Emotional disturbances & 47.04 & 30.20 & 34.02 & 0.004 \\
\hline Social life & 22.65 & 9.35 & 14.45 & 0.19 \\
\hline Personal life & 41.40 & 16.66 & 14.06 & 0.005 \\
\hline Sexual problems & 39.06 & 36.71 & 26.56 & 0.156 \\
\hline Cosmetic problems & 23.43 & 9.89 & 9.63 & 0.005 \\
\hline \multicolumn{5}{|l|}{ SELENIUM GROUP } \\
\hline Hypethyroidism symptoms & 23.71 & 19.11 & 16.36 & 0.03 \\
\hline Hypothyroidism symptoms & 29.41 & 16.54 & 15.44 & 0.002 \\
\hline Eye symptoms & 13.60 & 12.86 & 8.27 & 0.04 \\
\hline Fatigue & 46.63 & 40.75 & 42.43 & 0.136 \\
\hline Cognitive problems & 33.32 & 20.09 & 25.24 & 0.024 \\
\hline Anxiety & 42.40 & 27.69 & 31.12 & 0.013 \\
\hline Depression & 44.74 & 34.66 & 35.92 & 0.020 \\
\hline Emotional disturbances & 41.17 & 32.18 & 34.15 & 0.014 \\
\hline Social life & 12.86 & 4.77 & 4.77 & 0.067 \\
\hline Personal life & 19.36 & 9.80 & 6.61 & 0.013 \\
\hline Sexual problems & 30.88 & 27.94 & 25.73 & 0.52 \\
\hline Cosmetic problems & 8.08 & 3.67 & 2.45 & 0.072 \\
\hline
\end{tabular}

${ }^{*}$ Friedman test was applied to calculate the statistical significance of the difference of the scores between each visit

tibodies [3]. Some studies which investigated the correlation between Selenium supplementation and anti-thryoid antibody activity did not produce any conclusive results. $[8,9]$. Our study showed a decrease in antbody activity, but this decrease was found not only in the treated groups, but also in the control (Placebo) group, therefore we could not find a clear causality between Selenium supplementation and antibody serum levels. Another meta-analysis published in literature showed there was a moderate decrease in antibody levels and a small improvement in patients quality of life after three months Selenium supplementation in chronic autoimmune thyroidits patients [10]. A study which also investigated the effect of Selenium supplementation in Hashimoto thyroidits concluded that the decreasing of antibody levels was significantly higher in patients who had the initial levels of TPOAb levels very high (before starting the Selenium treatment) compared to those with low-moderate serum levels. Therefore patients with very high TPOAb serum levels could have more benefits from Selenium supplementation therapy than patients with lower antibody levels $[11,12]$. Also another factor which could be important for a bigger effect of Selenium therapy would be selecting patients who have a Selenium deficiency, by measuring Selenium serum levels [13].

The health-related quality of life, in our case specific to thyroid-diseases, is a subject in permanent debate in the current medical-field research. There is a tendency to ignore this subject in day to day medical practice because of the focus on somatic and objective features of diseases, but the outcome of the therapeutic act would be greatly 


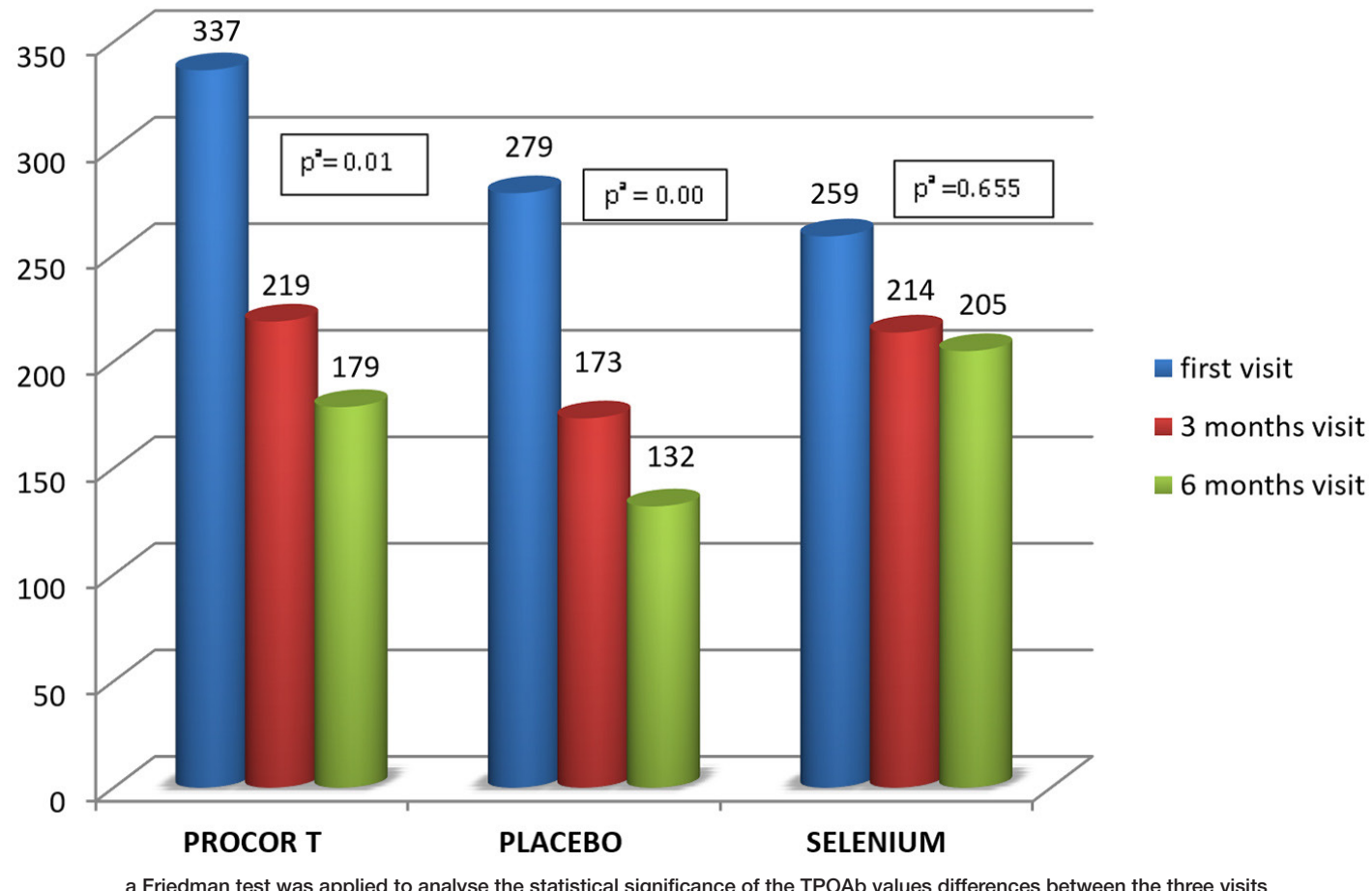

a Friedman test was applied to analyse the statistical significance of the TPOAb values differences between the three

Fig. 1. Mean values of TPOAb levels (IU/ml) for all three treatment groups on each visit.

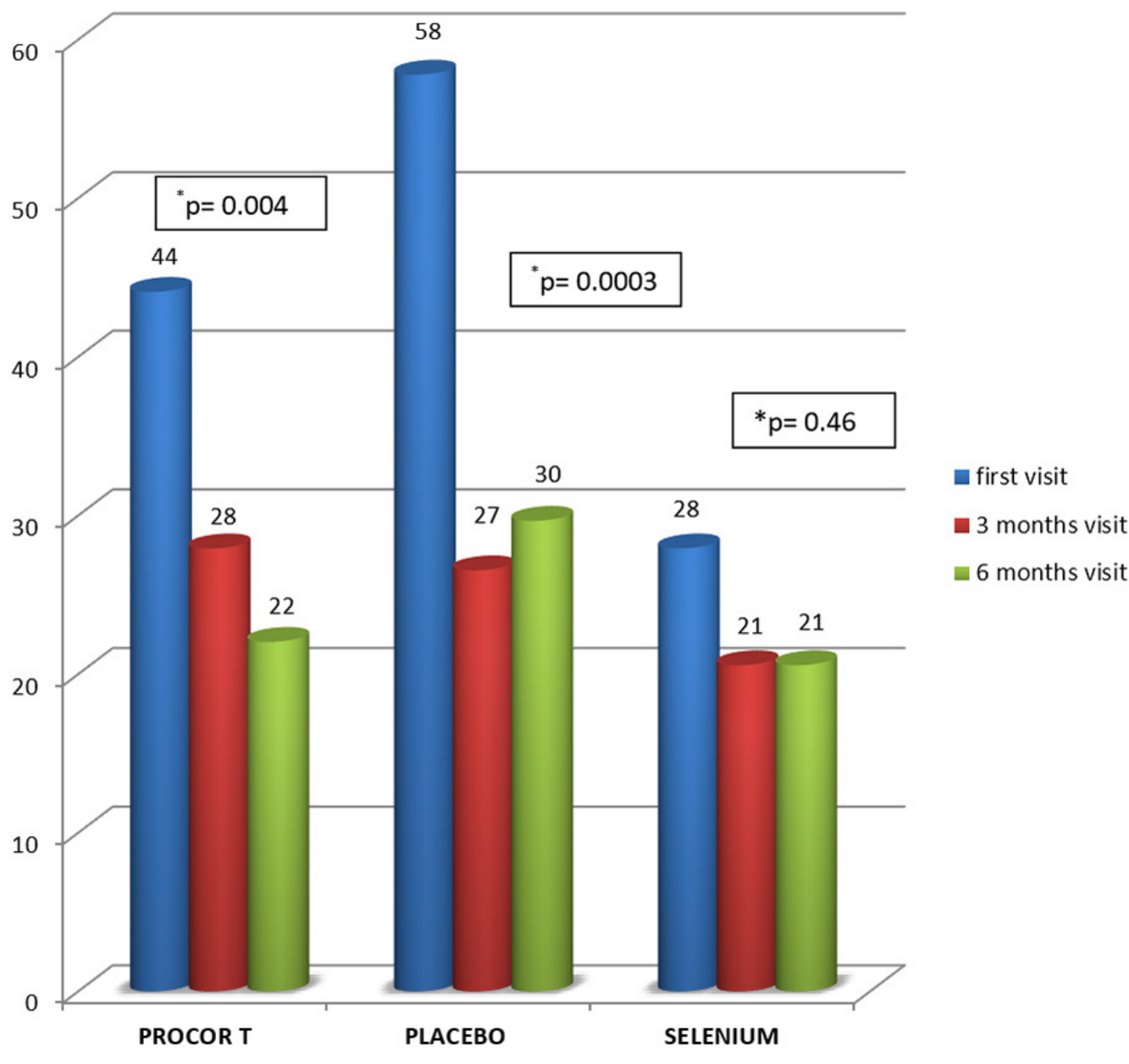

a Friedman test was applied to analyse the statistical significance of the score differences between the three visits

Fig. 2. THYPROro mean scores on the last question regarding the general impact on quality of life. 


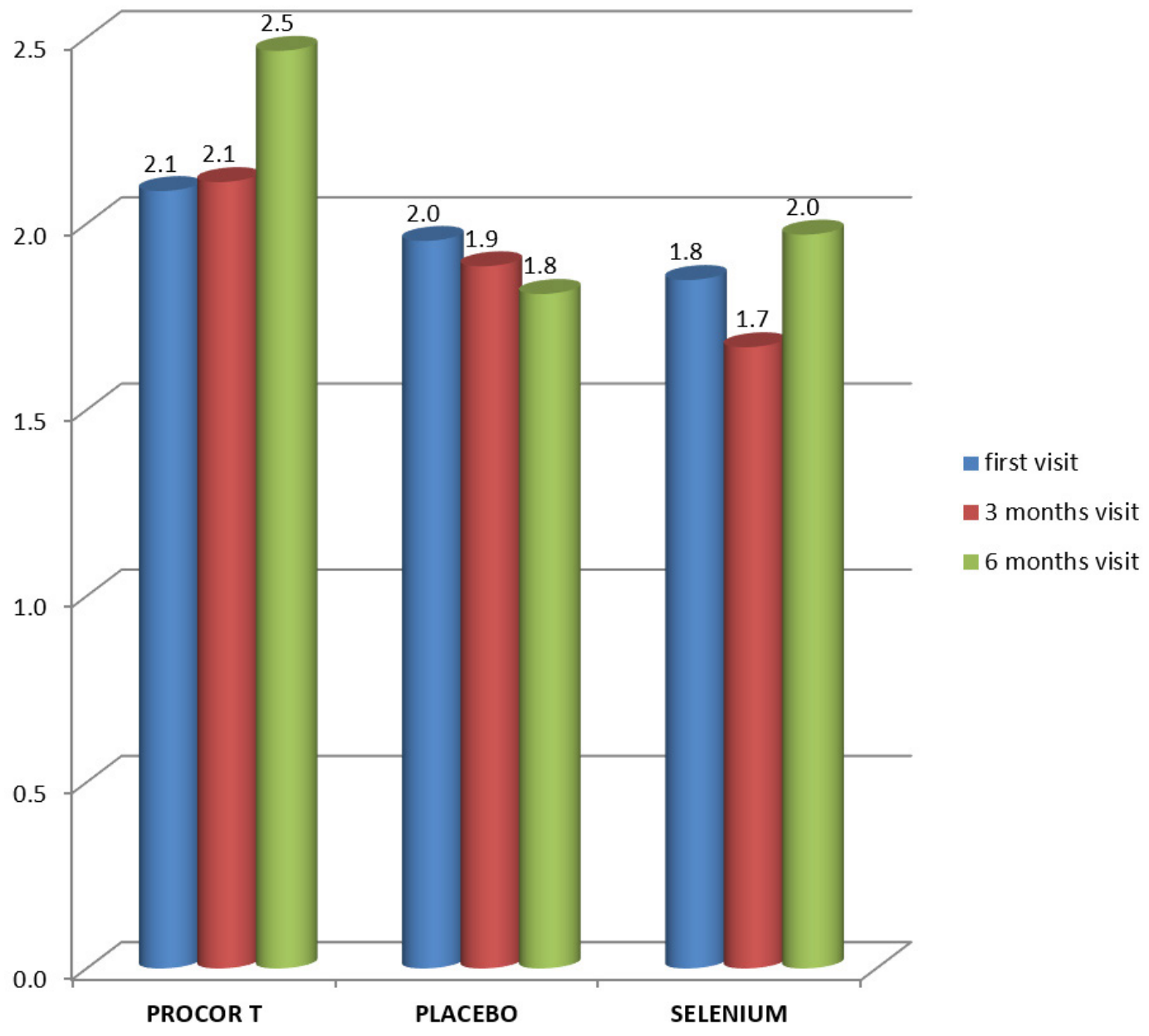

Fig. 3. Mean values of TSH $(\mu \mathrm{lU} / \mathrm{ml})$ for each treatment group on each visit.

improved by trying to include the quality of life aspects in the therapeutical management of patients, especially in those with chronic autoimmune diseases, like Hashimoto thyroiditis. A study from Italy evaluated the quality of life impairments in patients with various chronic thyroid diseases. An interesting finding of this study is that the quality of life in patients with autoimmune thyroiditis with normal thyroid function was significantly lower than in patients with other thyroid diseases, who presented subclinical hypo- or hyperthyroidism [14]. In our study we used the standard and validated unit called THYPROro (described in the „Methods" section of the article) as a tool to measure the health-related quality of life. The results showed a statistically significant improvement in most scales of the questionnaire for the Placebo group, with the exception of social and sexual life. In the Selenium group the results were similar to the Placebo group, with the scales showing no improvement being: cosmetic problems. social and sexual life. For the Procor T group patients showed improvements in the THYPROro scores as well, the exceptions being: depression, cognitive problems, cosmetic problems, social and sexual life. By analysing these results we could not find any relevat conclusion from our study regarding a benefit on the quality-of-life of these two Selenium-based supplements in patients with autoimmune thyroidits, as there was no statistically significant difference between the placebo and treated groups in any of the scales. We noticed that aspects related to sexual and social life had no significant changes between visits, therefore these might not be influenced by the autoimmune thyroid disease evolution too much. On the last question of the questionnaire regarding the impact of the quality of life in general, we noticed a statistically significant improvement in the score for the Procor T and Placebo groups only, and none for the Selenium group. Our findings show that there was a corellation between high TPOAb serum levels and a decreased quality of life. Other studies in literature have demonstrated there was a statistically significant corellation between high anti-thryoid antibody levels and an impaired quality of life, but no corellation between antibody levels and thyroid dysfunction [15]. These findings might indicate that the mechanisms through which the autoimmune disease affects the quality of life is not only related to the dysfunction of the thyroid. Many other insufficiently known physiopathological consquences of the autoimmune process in the human body might be responsible for the various symptoms that cause patients to feel a decline in the quality of life.

Our study was a preliminary one with multiple limitations, but potential for improvement and continuity. First 
of all, the number of patients included was fairly low (50), out of whom only 44 had positive TPOAb levels (the others having positive anti-thyroglobuline levels). Another limitation is due to the fact that the initial TPOAb values range was high, as both patients with very high antibody levels (above $500 \mathrm{UI} / \mathrm{ml}$ ) and patients with just mild positive levels were included. As the number of patients included was low, other limitations of our study could be noticed in several other aspects: BMI (Body Mass Index)$54 \%$ of the patients included presented a BMI over 25, so even though overweight and obesity is common in patients with autoimmune thyroidits, data regarding quality of life might have been influenced by this high percentage of obese or overweight patients. As it is known that associated diseases and medication could have a high impact on a patient's quality of life, we respected the inclusion criteria by not including patients with severe enough commorbities that would influence the quality of life results. For a more accurate investigation in future studies, the serum levels of Selenium should also be investigated before including the patients in the study, because Selenium defficiency could be a major factor that contributes to a higher benefit of Selenium-supplementation therapy in autoimmune thyroiditis patients. For a better compliance from the patients and a more facile gathering of data from the questionnaire, we recommend implementing an electronic version of the questionnaire, as data from recent studies indicated this could improve the process of researching the health-related quality of life aspects [16].

\section{Conclusions}

By analysing the results of this preliminary study we could conclude the following:

The two Selenium-based supplements which we studied did not prove to have any benefits regarding an improvement in the quality of life aspects.

Our study did not show a certain influence of the Selenium-based supplements on the TPOAb serum levels.

Most patients with autoimmune thyroidits included had a significant improvement in most aspects of quality of life between the entry - and 6 months follow-up visit, therefore the placebo-effect on some of these symptoms could have been beneficial.

The high antibody serum levels seem to be correlated to impaired quality of life, although the mechanism of this relation is not yet known, as none of the patients had any thyroid dysfunction during the study.

\section{Acknowledgements}

This research was supported by Sun Wave Pharma company. We thank them for the assistance and help provided for conducting this study.

\section{Authors' contribution}

Maximilian Cosma Gliga (Conceptualization; Data curation; Formal analysis; Funding acquisition; Investigation; Methodology; Project administration; Resources; Software; Supervision; Validation; Visualization; Writing original draft; Writing -review \& editing)

Ionela Maria Pascanu (Conceptualization; Formal analysis; Investigation; Project administration; Supervision; Validation; Visualization)

Camelia Gliga (Conceptualization; Data curation; Formal analysis; Funding acquisition; Investigation; Supervision; Validation; Visualization; Writing - review \& editing) Ancuta Elena Zahan (Conceptualization; Formal analysis; Investigation; Methodology; Software; Supervision; Validation; Visualization)

Iulian Merlan (Data curation; Formal analysis; Software; Visualization)

\section{References}

1. Van Zuuren EJ, Albusta AY, Fedorowicz Z, et al- Selenium supplementation for Hashimoto thyroiditis, Cochrane Database Syst Rev. 2013; Jun 6: 55 60.

2. Yalcin MM, Altinova AE, Cavnar $B$ et al- Is thyroid autoimmunity itself associated with psychological well-being in euthyroid Hashimoto thyroiditis?, Endocr J. 2017; 64(4): 425-429.

3. McGregor B- The Role of Selenium in Thyroid Autoimmunity: A Review, Journal of Restorative Medicine. 2015; 4: 83.

4. Schweizer U, Fradejas-Villar N- Why 21? The significance of selenoproteins for human health revealed by inborn errors of metabolism, FASEB J. 2018; 30(11): 3669-3681.

5. Schonburg L, Schweizer U, Kohrle J- Selenium and selenoproteins in mammals: extraordinary, essential, enigmatic, Cell Mol Life Sci. 2004; 61(16):1988-55

6. Watt $T$, Cramon $P$, Hegedüs $L$ et al- The thyroid-related quality of life measure ThyPRO has good responsiveness and ability to detect relevant treatment effects, J Clin. Endocrinol Metab. 2014; 99(10): 3708-14.

7. Zahan AE, Watt T, Pascanu I et al- The Romanian Version of the ThyroidRelated Patient-Reported Outcomes Thypro and Thypro-39. Translation and Assessment of Reliability and Crosscultural Validity, Acta Endo (Buc). 2018; 14(2): 192-200.

8. Liontiris MI, Mazokopakis EE- A concise review of Hashimoto thyroiditis $(\mathrm{HT})$ and the importance of iodine, selenium, vitamin D and gluten on the autoimmunity and dietary management of HT patients. Points that need more investigation, Hell J Nucl Med. 2017; 20(1): 51-56.

9. Vasiliu I, Preda C, Serban IL et al- Selenium status in autoimmune thyroiditis, Rev Med Chir Soc Med Nat lasi. 2015;119(4): 1037-44.

10. Yaofu F, Shuhang $X$, Huifeng $Z$ et al- Selenium Supplementation for Autoimmune Thyroiditis: A Systematic Review and Meta-Analysis, Int J Endocrinol. 2014; 2014: 231-234.

11. Gartner R, Gasnier $\mathrm{BCH}$ - Selenium in the treatment of autoimmune thyroidits, Biofactors. 2003; 19(3-4): 165-170.

12. Turker O, Kumanlioglu K, Karapolat I et al- Selenium treatment in autoimmune thyroidits: 9 month follow-up with variable doses, J Endocrinolog. 2006; 190(1): 151-156.

13. Duntas LH- The role of lodine and Selenium in Autoimmune Thyroiditis, HormMetab Res. 2015; Sep;47(10):721-6

14. Bianchi GP Zaccheroni V, Solaroli E et al- Health related quality of life in patients with thyroid disorders. A study based on Short-Form 36 and Nottingham Health Profile Questionnaires, Qual Life Res. 2004; 13(1): 45-54.

15. Bektas Uysal $H$, Ayhan M- Autoimmunity affects health-related quality of life in patients with Hashimoto's thyroiditis, Kaohsiung J Med Sci. 2016; 32(8): 427-33.

16. Rasmussen SL, Rejnmark L, Ebbehøj E et al- High Level of Agreement between Electronic and Paper Mode of Administration of a ThyroidSpecific Patient-Reported Outcome, ThyPRO, Eur Thyroid J. 2016; 5(1): 65-72. 